\title{
Localized fluidity modes and the topology of the constant-potential-energy hypersurfaces of Lennard-Jones matter
}

\section{Cotterill, Rodney M J; Madsen, J.}

\section{Published in:}

Physical Review B

Link to article, DOI:

10.1103/PhysRevB.33.262

Publication date:

1986

Document Version

Publisher's PDF, also known as Version of record

Link back to DTU Orbit

Citation (APA):

Cotterill, R. M. J., \& Madsen, J. (1986). Localized fluidity modes and the topology of the constant-potentialenergy hypersurfaces of Lennard-Jones matter. Physical Review B, 33(1), 262-268.

https://doi.org/10.1103/PhysRevB.33.262

\section{General rights}

Copyright and moral rights for the publications made accessible in the public portal are retained by the authors and/or other copyright owners and it is a condition of accessing publications that users recognise and abide by the legal requirements associated with these rights.

- Users may download and print one copy of any publication from the public portal for the purpose of private study or research.

- You may not further distribute the material or use it for any profit-making activity or commercial gain

- You may freely distribute the URL identifying the publication in the public portal 


\title{
Localized fluidity modes and the topology of the constant-potential-energy hypersurfaces of Lennard-Jones matter
}

\author{
R. M. J. Cotterill and J. U. Madsen \\ Department of Structural Properties of Materials, The Technical University of Denmark, Building 307, DK-2800 Lyngby, Denmark
}

(Received 12 August 1985)

\begin{abstract}
Sections of configuration space for Lennard-Jones matter were obtained by probing all the normal-mode energy profiles, following diagonalization of the dynamical matrix for a 240-particle system. For the crystal and sufficiently cold glass, these are single welled, whereas increasing numbers of double wells occur as the glass is warmed toward the fluid. This indicates that there might be a fundamental difference between the topologies of the constant-potential-energy hypersurfaces of crystalline and noncrystalline Lennard-Jones matter.
\end{abstract}

\section{INTRODUCTION}

We have made observations which indicate that there might be an interesting distinction between the crystalline and noncrystalline (i.e., glassy, liquid, and gaseous) states of Lennard-Jones matter. This distinction would be independent of any identification of specific atomic arrangements, such as special distributions of free volume in pseudorandom packings of atoms or defects in quasicrystalline configurations. We have formally applied the mechanism of diagonalization of the dynamical matrix to instantaneous configurations of our model. We probed the normal-mode energy profiles to obtain onedimensional configuration space sections, and found these to possess only single wells in the crystalline state. In the noncrystalline states, however, some of the sections displayed double-well behavior. These double wells, which occur more frequently as the glass is warmed towards the fluid state, are indicative of a fundamental difference between the topologies of the constantpotential-energy hypersurfaces of crystalline and noncrystalline Lennard-Jones matter. If this difference is subsequently shown to be generally applicable to matter with other types of interatomic force, it could prove to be one of the defining characteristics of the noncrystalline state.

The available memory size in some of the latest digital computers is now sufficiently large to permit calculation of all the vibrational eigenmodes in systems of several hundred particles. There has been an understandable interest in identifying these modes in the various physical states of matter, and the ingenious quench-echo technique, ${ }^{1,2}$ in particular, has shed much light on vibrations in glass. We were interested in all the modes of our three-dimensional 240-particle system, in its various physical states, both those with positive eigenvalues, which correspond to real vibrational frequencies, and the imaginary-frequency negative eigenvalue modes. The latter are not accessible through the quench-echo approach, so we diagonalized the entire dynamical matrix, of order 720, a task which takes about $30 \mathrm{~min}$ on an IBM 3083 computer. We chose to simulate a system with 240 atoms because the corresponding number of degrees of freedom (i.e., 720) is close to the maximum that could be handled by the matrix diagonalization procedure, without running into prohibitive round-off problems, and also because this system provides a simple geometrical relationship to the 336-atom model we have reported on previous$1 y .^{3-5}$

Details of the computer simulations are presented in Sec. II, including a definition of the reduced units which were used throughout the work. Section III describes the main results of the study, namely, those appertaining to the topology of configuration hyperspace for the various physical states investigated. We were also interested in the degree of localization of the different eigenmodes corresponding to each state, and results which shed light on this question are presented in Sec. IV. Finally, and reflecting our continuing interest in the entropy changes which accompany the transitions between the different states, ${ }^{3,4}$ we have investigated the relationship between the entropy and the diffusion coefficient as the system is warmed from the glassy state into the liquid. These results are discussed in Sec. V. Section VI summarizes the new information gleaned from all these studies and puts them in perspective, both in general, with respect to current knowledge of the nature of the different states of simple matter and the transitions between them, and, in particular, to some other recent studies which have addressed themselves to the same broad questions. ${ }^{6,7}$

\section{DYNAMICAL MODEL}

The study of molecular model systems, typically comprising a few hundred particles, is now a standard and well-documented $^{8}$ accomplishment of modern computational physics. For simple substances, such as the noble gases, the assumption of purely central forces has usually been taken as justified, and the most-used functional form is that proposed by Lennard-Jones, namely,

$$
V_{\mathrm{LJ}}\left(r_{i j}\right)=\epsilon\left[\left(r_{0} / r_{i j}\right)^{12}-2\left(r_{0} / r_{i j}\right)^{6}\right],
$$

where $V_{\mathrm{LJ}}\left(r_{i j}\right)$ is the potential energy between the two particles $i$ and $j$, separated by the distance $r_{i j}$. This interaction was cut off at the distance $2.12 r_{0}$, a truncation 
which allows each atom to interact with its closest 54 neighboring atoms in the low-temperature crystal, and approximately that same number in all the states investigated. The constants, $\epsilon$ and $r_{0}$, in the interaction potential were used to define the reduced units of density, $r_{0}^{-3}$; energy, $\epsilon$; pressure, $\epsilon / r_{0}^{3}$; and temperature, $\epsilon / k_{B}, k_{B}$ being Boltzmann's constant. The reduced units for the diffusion coefficient, $D$, and the entropy, $S$, were thus $r_{0}(\epsilon / M)^{1 / 2}$ and $k_{B}$, respectively, where $M$ is the atomic mass. It is further convenient to use a reduced system in which the latter is set at unity.

The three-dimensional system of $N$ particles was studied by the technique of molecular dynamics, ${ }^{8}$ which is essentially a finite difference approach to the solution of the Newtonian equations of motion:

$$
\ddot{\mathbf{r}}=-\nabla \Phi(\mathbf{r})
$$

where $\Phi(\mathbf{r})$ is the total potential energy of the system when its configuration is specified by the $3 N$-dimensional vector $\mathbf{r}$. The reduced computational time step was $3 \times 10^{-3}$, in units of $r_{0}(M / \epsilon)^{1 / 2}$. The model was made pseudoinfinite by use of periodic boundary conditions, the irreducible computational cell having the dimensions $5 r_{0}$ by $4(3)^{1 / 2} r_{0}$ by $2(6)^{1 / 2} r_{0}$. We were particularly interested in the topology of constant potential-energy surfaces of $\Phi(\mathbf{r})$ in $3 N$-dimensional hyperspace, and we probed sections of this function by diagonalizing the dynamical matrix, of order $3 N$, the elements of which are $\partial^{2} \Phi / \partial \mathbf{x}_{i, m} \partial \mathbf{x}_{j, n}$. The subscripts $m$ and $n$ each denote one of the three Cartesian positional coordinates.

Although the limitation of the interparticle interaction did produce numerous zero-valued matrix elements, the truncation was not so tight as to produce a truly sparse matrix. We were thus dependent upon one of the standard diagonalization procedures, and it proved convenient to use the EIGRS routine of the IMSL (International Mathematical and Statistical Libraries) system. ${ }^{9}$ Even using double precision it was not possible to successfully diagonalize a matrix of order $1008(=3 \times 336)$, and this precluded using the 336-particle model we have studied earlier. $^{3-5}$ It was possible to diagonalize a 720-order matrix, however, and the 240-particle system was in fact obtained by slicing away a 96-particle slab from the largest dimension of our earlier model, retaining the original particle density.

\section{POTENTIAL-ENERGY HYPERSPACE SECTIONS}

The system of particles was simulated in the crystalline, glassy, and fluid states, all runs being carried out at the same density to facilitate comparison with previous work,,$^{3-5}$ namely, a density of unity in our reduced units. After proper equilibration of a given state, the instantaneous atomic configuration was used as input for the dynamical-matrix diagonalization. In general, this was found to produce both positive and negative eigenvalues, and we paid particular attention to the negative members, which correspond to imaginary eigenfrequencies. Each eigenvector $\overline{\mathbf{A}}_{p}$, given by $\overline{\mathbf{A}}_{p}=\left(a_{p q}\right)$, with $p, q$ $=1,2, \ldots, 720$, defines a unique direction in 720 dimensional configuration space. We probed all of the system's energy profiles $E_{p}(\lambda)=\Phi\left(\overline{\mathbf{R}}_{0}+\lambda \overline{\mathbf{A}}_{p}\right)-\Phi\left(\overline{\mathbf{R}}_{0}\right)$,

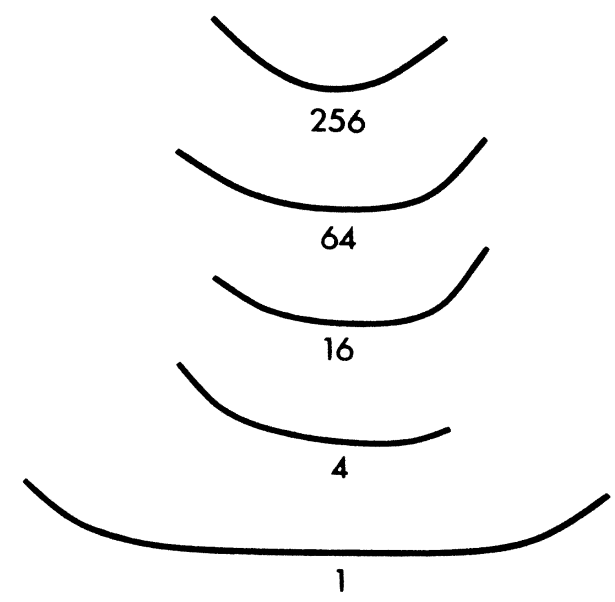

FIG. 1. Typical normal-mode energy profiles, arbitrarily selected with mode numbers mutually separated by a factor of 4 , for the crystal at a reduced temperature of 0.670 . The abscissa represents distance in the direction of the eigenvector, and the ordinate shows the relative energy. The width of the lowest profile corresponds to $|\lambda A|=0.50$, and the linearly-plotted energy has been arbitrarily cut off where the next computational point would carry the (now rapidly rising) energy above 0.17 . Figures 2 and 3 use the same scales. The eigenvalues of the five states shown corresponded to squared frequencies of $-108.5,-51.3$, $-18.2,19.0$, and 194.8, respectively, in increasing order.

where $\overline{\mathbf{R}}_{0}$ is the 720 -dimensional state vector of the above instantaneous reference configuration and $\Phi\left(\overline{\mathbf{R}}_{0}\right)$ is the total interaction energy. This was done for $\left|\lambda \overline{\mathbf{A}}_{p}\right|$ values up to about the nearest-neighbor distance. These energy profiles displayed marked differences depending upon the state under investigation. The simplest type had a minimum at $\lambda=0$, rising monotonically for both positive and negative $\lambda$ values. The more general profile had a

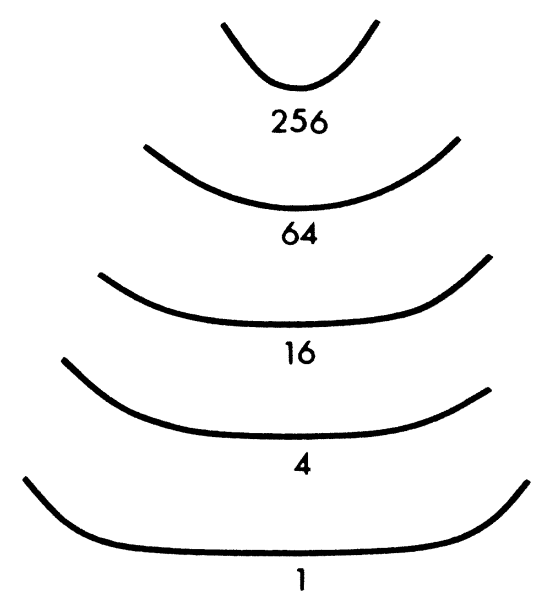

FIG. 2. Typical normal-mode energy profiles for the glass at a reduced temperature 0.034 . The abscissa represents distance in the direction of the eigenvector, and the ordinate shows the relative energy. The scales are the same as in Fig. 1. The eigenvalues for the five states shown corresponded to squared frequencies of $-15.8,-1.7,7.7,48.0$, and 227.1 , respectively, in increasing order. 


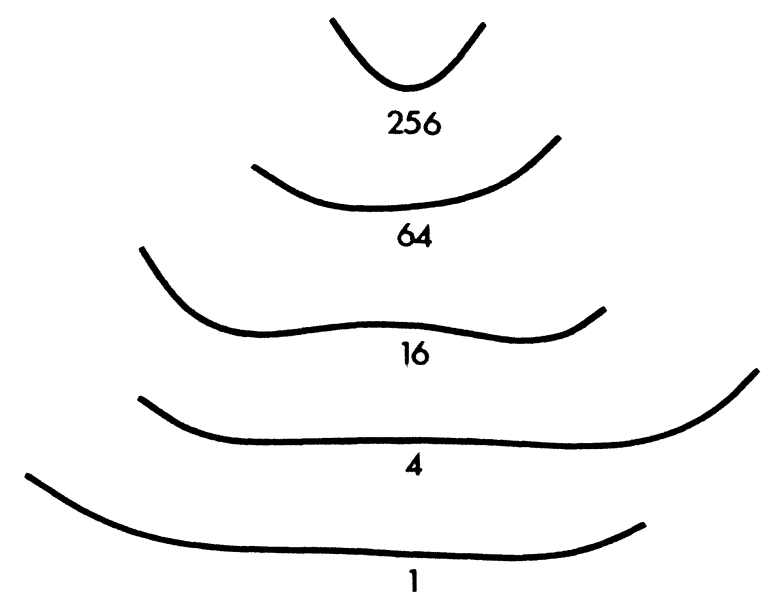

FIG. 3. Typical normal-mode energy profiles for the fluid at a reduced temperature of 0.405 . The abscissa represents distance in the direction of the eigenvector, and the ordinate shows the relative energy. The scales are the same as in Fig. 1. The eigenvalues for the five states shown corresponded to squared frequencies of $-113.3,-68.8,-39.9,5.0$, and 210.2 , respectively, in increasing order. In contrast to the situation in the (warm) crystal and (cold) glass, shown in Figs. 1 and 2, respectively, some of the lower profiles display double-well behavior.

minimum at finite $\lambda$, either positive or negative, but for this type too, $\partial E_{p}(\lambda) / \partial \lambda$ was zero only at that single minimum. In both the crystal over the entire range from zero temperature to the previously-reported crystal instability, ${ }^{3}$ and the glass around zero temperature, only these types of profiles were observed. Figure 1 shows a selection of typical profiles for the crystalline state, the numbers identifying the modes (in increasing order starting from the most negative eigenvalue of the entire 720member set). Figure 2 shows a similar selection for a low-temperature glass.

As the glass was warmed towards the fluid state, we observed progressively more profiles of a quite different type: profiles with $\partial E_{p}(\lambda) / \partial \lambda=0$ for three different values of $\lambda$, one positive, one at zero, and one negative. Figure 3 shows typical profiles of this double-well type. For such modes, the system is unstable against spontane-

TABLE I. The number of double-welled normal-mode energy profiles, $N_{d}$, as a function of the reduced temperature, $T$, for the amorphous states (i.e., glass to fluid). Also shown are the excess entropy relative to the crystalline state at the same temperature, $\Delta S$ (for the analogous 336-particle system; see Fig. 8), and the diffusion coefficient, $D$, both in the reduced units described in Sec. III.

\begin{tabular}{cccc}
\hline \hline$T$ & $N_{d}$ & $\Delta S$ & $D$ \\
\hline 0.034 & 0 & 0.00 & 0.000 \\
0.195 & 4 & 0.10 & 0.014 \\
0.293 & 7 & 0.18 & 0.018 \\
0.405 & 9 & 0.25 & 0.023 \\
0.606 & 16 & 0.35 & 0.028 \\
0.831 & 38 & 0.44 & 0.077 \\
1.392 & 59 & 0.57 & 0.168 \\
\hline \hline
\end{tabular}

ous atomic rearrangement along the corresponding eigenvector. The number of observed double-well profiles is listed as a function of reduced temperature in Table $I$. These double wells are indicative of a fundamental difference between the topologies of the constant-potentialenergy hypersurfaces of crystalline and noncrystalline matter.

\section{EIGENMODE LOCALIZATION}

If a small model such as ours is to establish its viability, it is clear that it must display all of the significant behavior expected in a system of macroscopic dimensions. There will, of course, be features that are a direct consequence of the model's finite size and of the use of periodic boundary conditions. The first of these factors introduces the familiar temperature variations, for example, while the periodic boundaries imply a long-wavelength cutoff of the phonons. Our main concern, however, was with the presence of all modes that might have an important bearing on the transitions between the various physical states. We thus checked the spatial extent of the most pronounced instability modes, for each state. This was conveniently done by stereo plotting of the various eigenvectors.

By way of introduction to the method of plotting, we reproduce in Fig. 4 two of the 720 eigenvectors for one of the crystalline states. The instantaneous atomic positions are indicated by the dots, upon which all of the components of the eigenvector have been superimposed as lines, with lengths proportional to the magnitudes of the components. The lower stereo plot shows eigenvector number 8, and the plot is instantly interpretable as displaying a single transverse wave in the vertical direction, with one wavelength fitted into the irreducible cell's vertical dimension. The upper stereo pair of Fig. 4, which shows eigenvector number 60 for the same state, is not nearly so easy to rationalize; the complexity stems from the mixture of several spatial harmonic waves, having various wavelengths, directions, transverse and/or longitudinal characters, and phases. Both the eigenvectors shown in Fig. 4 would be said to be delocalized, and this was true of all eigenvectors for all the crystalline states investigated.

Eigenvectors number 1, 100, and 700, for the glassy state at a reduced temperature of 0.034 , are shown as stereo pairs in the lower, middle, and upper pictures of Fig. 5, respectively. The degree of localization was found to vary slowly and systematically upon proceeding up through the entire set of eigenvectors, and these three examples reveal that the highest and lowest extremes of the set are characterized by considerable localization. The majority of the eigenvectors lie between these extremes, however, and they display the delocalization illustrated for eigenvector number 100 .

Figure 6 corresponds to the fluid state at a reduced temperature of 0.405 . Once again, and to facilitate comparison with Fig. 5, eigenvectors number 1, 100, and 700 have been plotted, as stereo pairs. As for the glass, the extreme members of the set show considerable localization, whereas the central members are delocalized. Figures 5 

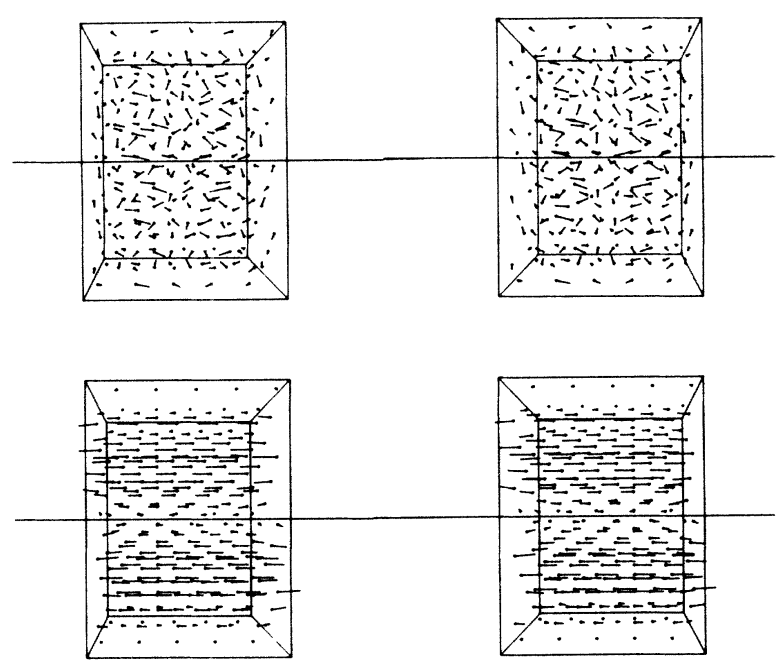

FIG. 4. Stereo plots showing the instantaneous atomic positions (as dots), with the eigenvectors superimposed (as lines), of two modes for the crystal at a temperature of 0.00 . The box indicates the positions of the periodic boundaries. The lower picture shows mode 8 , with squared eigenfrequency 26.3 , while the upper picture corresponds to mode 60 , with squared eigenfrequency 112.8 .
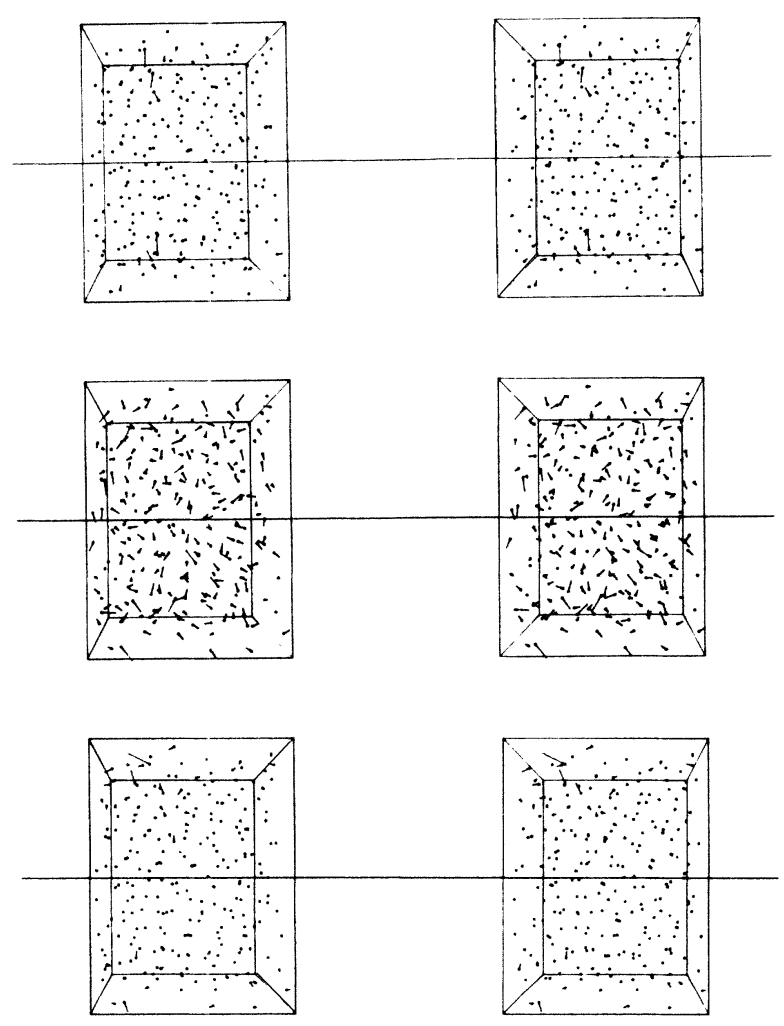

FIG. 5. Stereo plots showing the instantaneous atomic positions, with the eigenvectors superimposed, of three modes for the glass at a reduced temperature of 0.034 . The lower picture shows mode 1 , with squared eigenfrequency -15.8 , the middle picture shows mode 100 , with squared eigenfrequency 78.0 , and the upper picture shows mode 700 , with squared eigenfrequency 1517.9.
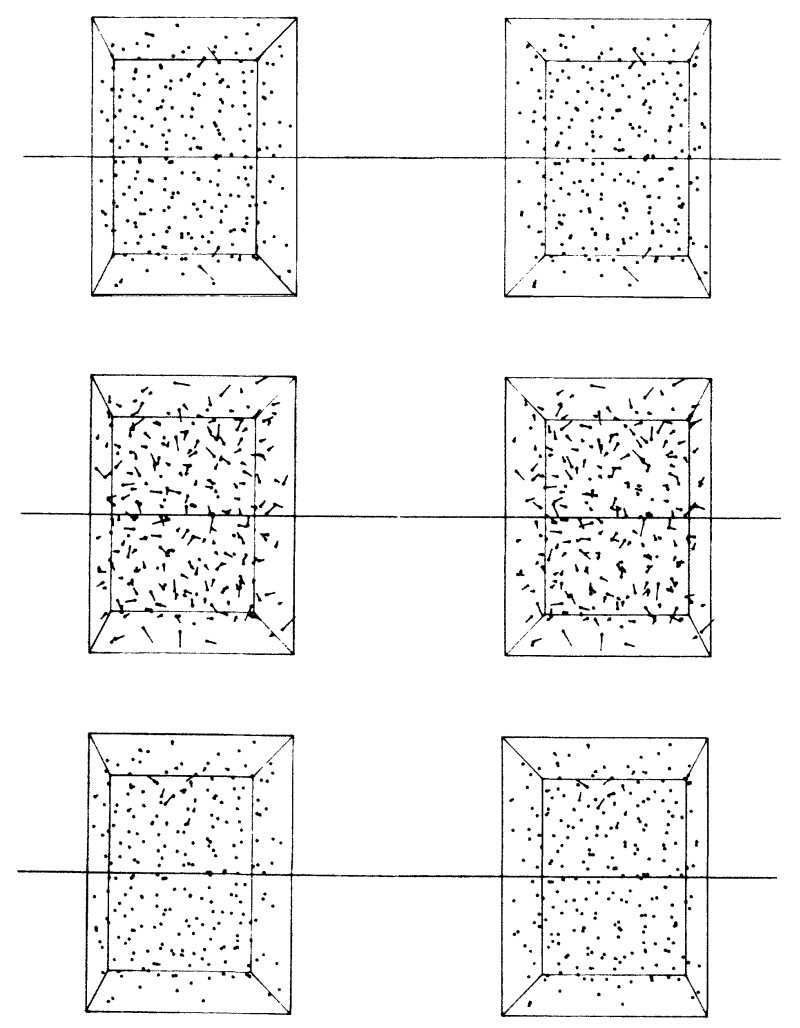

FIG. 6. Stereo plots showing the instantaneous atomic positions, with the eigenvectors superimposed, of three modes for the fluid at a reduced temperature of 0.405 . The lower picture shows mode 1 , with a squared eigenfrequency of -113.3 , the middle picture shows mode 100 , with squared eigenfrequency 34.2 , and the upper picture shows mode 700 , with squared eigenfrequency 2390.6 .

and 6 do indeed resemble each other, and one recalls the indication in our previous entropy study ${ }^{3}$ that the fluid and glassy states are simply different ranges of one continuous phase field. These plotted eigenvector trends appear to endorse that finding.

It is interesting to note the qualitative similarity between our observed localized modes and those predicted by Weisskopf. ${ }^{10}$ Although a full analysis demands a quantitative criterion, whereby only those atoms displaced through a distance in excess of some threshold are regarded as participating in a mode, for example, it could loosely be stated that approximately 10 to 20 atoms are involved in the nonoscillatory mode corresponding to eigenvector number 1 in Fig. 6. Weisskopf arrived at a similar number of atoms, through a calculation based on simple assumptions and using thermodynamic data.

\section{ENTROPY AND DIFFUSION COEFFICIENT}

It is interesting to evaluate the new results presented in Sec. III in the light of our earlier observation ${ }^{3}$ that the crystalline and glassy states, at the same density and (low) temperature, have approximately the same entropy, and our earlier suggestion ${ }^{3,4}$ that the excess entropy of the liquid, compared with the crystal at the same density and 


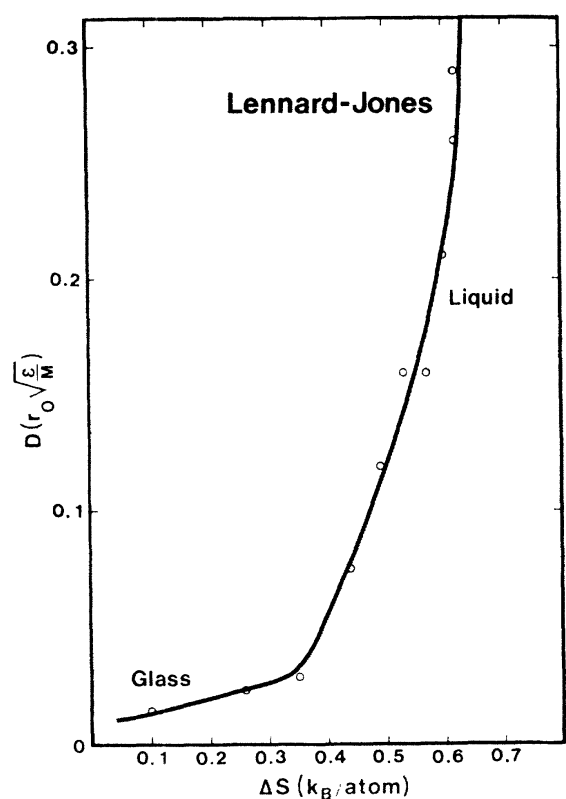

FIG. 7. Diffusion coefficient, $D$, as a function of the excess entropy of the glass fluid with respect to the crystal, $\Delta S$, both in the reduced units described in Sec. II.

temperature, must thus be due to the liquid's fluidity rather than its disorder. As a further check on this issue, we monitored the mean-square atomic displacement as a function of time, for each equilibrated state, and thereby measured the diffusion coefficient. As can be seen from the results shown in Fig. 7, in which the diffusion coefficient is plotted against the excess entropy of the noncrystalline state over the crystalline state at the same temperature, diffusion becomes prominent around the temperature at which the population of our new instability modes is increasing sharply. This endorses the suggested $\operatorname{link}^{4}$ between the fluid's acquisition of entropy, compared with the crystalline state, and the gradual replacement of vibrational modes by diffusive modes. For convenience during the discussion in Sec. VI, we reproduce here our earlier suggestions for the assignments of the constant-volume entropy difference between the different states. ${ }^{4}$ They are shown in Table II.

\section{DISCUSSION}

The object of this study was to throw new light on the nature of the various states of simple matter and their relationships with one another. If it were possible to determine the precise shape of the potential-energy hypersurface for the different states, and to overcome the considerable difficulty of interpreting its $3 \mathrm{~N}$-dimensional topology, these inter-relationships would no doubt become more transparent. Having access only to one-dimensional sections of the energy hypersurface, we obviously fall well short of that ideal, and it is important to appreciate the difficulties which arise from this limitation. The seemingly simple change from single-well to double-well behavior in some of the one-dimensional configuration space sections could imply a dramatic rise in the complexity of the constant-potential-energy hypersurfaces. The principles can be appreciated by considering the energy profiles of a two-dimensional subspace with a hat-shaped energy function, with an annular minimum. The local profiles along radial sections would have only one minimum, but profiles along sections at right angles to a radius might have one or two minima; the possibility of the state point running around the ring-shaped valley inside the hat's "brim" would not be apparent from onedimensional sections. By the same token, the occurrence of double wells in our monitored sections could indicate the existence both of labyrinthine (and multiply connected) constant-potential-energy hypersurfaces and diffusive modes that do not involve potential-energy barriers.

The latter suggestion is not in accord with the situation envisaged by Stillinger, DiMarzio, and Kornegay ${ }^{11}$ and recently advocated by Stillinger and Weber., ${ }^{6,7}$ In the former paper it was suggested that the salient feature of the energy hypersurface is its individual pockets interconnected by deep and narrow channels, and the latter work is also interpreted in terms of energy barriers between short-lived but mechanically stable packings of particles. If that were indeed the situation in a liquid, it would make that state not unlike a crystal gradually creeping due to the motion of vacancies. During the jump of a vacancy, ${ }^{12}$ one atom moves through a distance equal to that between adjacent atoms, while several of the adjacent atoms move through distances that are a few percent of this. Such motions do not, of course, lead to the collapse of the crystal's struc-

TABLE II. Relative entropies for the isochoric changes of state of simple substances, in the reduced units employed in this paper. The asterisk denotes the fact that short-wavelength transverse waves are present in the liquid. Their extent varies with element and this will probably influence the value of the liquid entropy.

\begin{tabular}{lcccc}
\hline \hline \multicolumn{1}{c}{ State } & $\begin{array}{c}\text { Number of } \\
\text { Longitudinal } \\
\text { oscillatory }\end{array}$ & $\begin{array}{c}\text { degrees of freedom per atom } \\
\text { Transverse } \\
\text { oscillatory }\end{array}$ & Diffusive & $\begin{array}{c}\text { Isochoric } \\
\text { entropy } \\
\text { relative to } \\
\text { crystal }\end{array}$ \\
\hline Ideal gas & 0 & 0 & 3 & 1.0 \\
Liquid* & 1 & $0^{*}$ & $2 *$ & $\sim 0.63$ \\
Glass & 1 & 2 & 0 & 0 \\
Crystal & 1 & 2 & 0 & 0 \\
\hline \hline
\end{tabular}


ture. The energy hypersurface for a crystal with vacancies must be quite similar to the picture presented by Stillinger, DiMarzio, and Kornegay, ${ }^{11}$ and the energy barrier for a vacancy jump has been measured for numerous substances. $^{12}$

Stillinger and Weber ${ }^{6}$ also report a mean energy barrier, having a height of 2.16 (in the same reduced units as those employed in this paper), for a system of 32 particles. It is important to note that this result was obtained by a quite different method than the one we have employed. Their steepest-descent approach is equivalent to an ultrarapid quench, and it is not surprising that this finds local minima of the system. The present study, on the other hand, reveals the probability that there are curved valleys which circumvent the barriers. Typical differences between the two wells and the intervening maximum in the onedimensional sections shown in Fig. 3 lie in a range which is a factor of nearly 50 below Stillinger and Weber's 2.16. The latter value might, however, deviate significantly from the barrier height characteristic for an infinite system.

An outstanding question regarding the glassy state is whether or not the change from glass to liquid should be regarded as a phase transition. Several aspects of both previously-reported work and the present studies indicate that the change is continuous. The entropy difference between the noncrystalline and crystalline states, at constant volume, is a smoothly increasing function of temperature, ${ }^{3}$ with no indication whatsoever of a sudden change of regime. (Figure 8 shows our earlier data for a 336particle Lennard-Jones system, and is reproduced here for the reader's convenience. We have yet to calculate the analogous data for the 240-particle system, but believe that it would resemble that shown in the figure.) Then again, the spatial extents of the eigenvectors for the different regions of the eigenvalue spectrum are similar in the glass and the liquid, as is apparent from a comparison of Figs. 5 and 6; this suggests an essential similarity between the two states. The approximate equality between the entropies of the glass and crystal, reported earlier, ${ }^{3}$ indicates that for both these states the state point is confined to a local potential-energy valley in configuration space. This view receives support from the similarity of the one-dimensional sections for these two states, as shown in Figs. 1 and 2. Our "hat" analogy is thus seen to be too symmetrical; the disorder of the glass probably implies that the hat is deformed, so as to break up the single valley into kidney-shaped depressions. The extension to many dimensions is, of course, difficult to envisage. At the lowest temperature, in the glass, the state point is confined to a local valley, but it might be able to gain access to an adjacent valley by traversing a relatively low energy barrier. This idea underlies the explanation of the linear temperature dependence of the specific heat of glasses at low temperature, as proposed by Anderson, Halperin, and Varma, ${ }^{13}$ and by Philips. ${ }^{14}$ With increasing temperature more and more valleys can be sampled, but this trend is gradual; the change is one of degree rather than type. It remains a moot point as to whether the change can be thought of in terms of the excitation of one two-level sys-

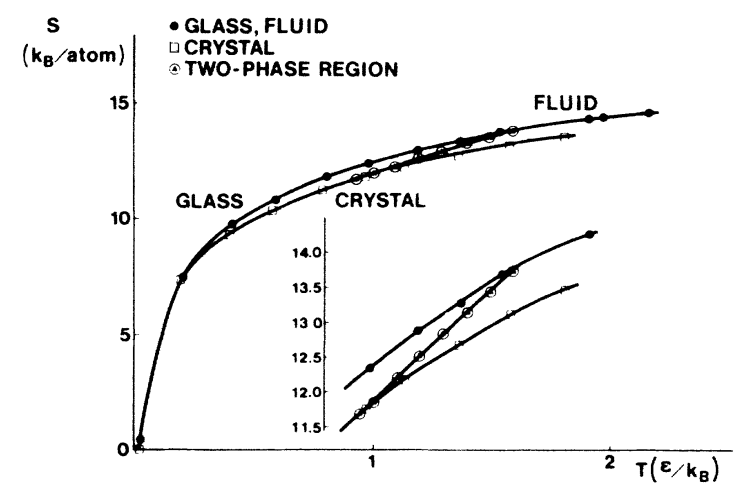

FIG. 8. Entropy of the crystalline and noncrystalline states, for a 336-particle Lennard-Jones system, relative to the crystal value at the reduced temperature of 0.017 . The inset shows the two-phase region with an expanded ordinate axis. The entropy difference between the glass and the crystal, at the same density, decreases to zero ( $\pm 0.05 k_{B}$ per atom) as zero temperature is approached.

tem provoking accesss to further two-level systems as recently invoked by Varma, Dynes, and Banavar. ${ }^{15}$

The reasonably well-defined knee in the curve of Fig. 7 is intriguing. Although our strongest inclination is to emphasize that this might be fortuitous, it must be admitted that it occurs at a rather suggestive value of entropy, namely, around 0.3. This is just about half of the value 0.63 which we have attributed to disappearance of two of the three vibrational modes, namely, the two transverse modes, on passing from the glass to the liquid (see Table II and the discussion in Sec. V). If the approximate value of 0.3 has any significance, it could be interpreted as indicating that just one transverse mode, on average, disappears as the glass starts to pass into the liquid state. But it might be preferable to conclude that this applies more generally to $N$ of the $3 N$ total modes, and it could be related to the change of regime from confinement of the state point to an isolated hyperspace well to access to many such wells.

As noted in Sec. III, the one-dimensional sections for the crystal were only single-welled, for all temperatures up to the previously reported breakdown., In this case, the state point is always confined to a single closed region of hyperspace, and this is consistent with the conclusion that the crystalline state is invulnerable to thermal disturbance up to and beyond the melting point; that there is indeed nothing which could justifiably be referred to as premelting, either defect mediated or otherwise. A preliminary announcement of our findings can be found in Ref. 16.

\section{ACKNOWLEDGMENTS}

We are grateful to Sir Sam Edwards, Göran Grimwall, Volker Heine, Toshiyuki Ninomiya, Aneesur Rahman, and Victor Weisskopf for stimulating discussions, the NEUCC (Northern European University Computing Center) for helpful advice, and Statens Naturvidenskabelige Forskningsråd for support. 
${ }^{1}$ G. S. Crest, S. R. Nagel, and A. Rahman, Solid State Commun. 36, 875 (1980).

${ }^{2}$ G. S. Crest, S. R. Nagel, and A. Rahman, Phys. Rev. Lett. 49, 1271 (1982).

3J. U. Madsen and R. M. J. Cotterill, Phys. Lett. 83A, 219 (1981).

4J. U. Madsen and R. M. J. Cotterill, Phys. Scr. 24, 959 (1981).

${ }^{5}$ R. M. J. Cotterill and J. U. Madsen, Nature 288, 467 (1980).

${ }^{6}$ F. H. Stillinger and T. A. Weber, Phys. Rev. A 28, 2408 (1983).

${ }^{7}$ F. H. Stillinger and T. A. Weber, J. Chem. Phys. 80, 4434 (1984).

${ }^{8}$ R. W. Hockney and J. W. Eastwood, Computer Simulations Using Particles (McGraw-Hill, New York, 1981).

${ }^{9}$ International Mathematical and Statistical Libraries Reference
Manual, 9th ed. (IMSL, Houston, 1982), Vol. 2.

10V. F. Weisskopf, Trans. N.Y. Acad. Sci. 38, 202 (1977).

${ }^{11}$ F. H. Stillinger, E. A. DiMarzio, and R. I. Kornegay, J. Chem. Phys. 40, 1564 (1964).

${ }^{12}$ A. C. Damask and G. J. Dienes, Point Defects in Metals (Gordon and Breach, New York, 1964).

${ }^{13}$ P. W. Anderson, B. I. Halperin, and C. M. Varma, Philos. Mag. 25, 1 (1972).

${ }^{14}$ W. A. Phillips, J. Low Temp. Phys. 7, 351 (1972).

${ }^{15}$ C. M. Varma, R. C. Dynes, and J. R. Banavar, J. Phys. C 15, L1221 (1982).

${ }^{16}$ R. M. J. Cotterill and J. U. Madsen, Bull. Am. Phys. Soc. 30, 123 (1985). 